\title{
Journal of World Architecture
}

\author{
Review Article
}

\section{Study on the Constructing Strategies of Rural Life Circles in Chinese Metropolitan Areas}

\author{
Dongshu $\mathrm{Li}^{1}$, Yueping $\mathrm{Hu}^{2 *}$, Darong Deng ${ }^{3}$ \\ ${ }^{1}$ China Academy of Urban Planning \& Design, Beijing 100044, China \\ ${ }^{2}$ Southwest Jiaotong University, Chengdu 611756, China \\ ${ }^{3}$ Chongqing Planning \& Designing Institute, Chongqing 401121, China
}

\begin{abstract}
Based on the aims of "issues of agriculture" and "three highs" in the new era, the metropolitan rural areas are targeted under the trend of counterurbanization. It follows the theory of Social Space Building, Maslow's Theory of Demand, and the theory of Social Governance. It mainly explores rural constructing strategies on development and the life with clear governance integration, range, hierarchy and main function as well as completing the issues such as ecology, culture, industry, living environment and livelihood facilities.
\end{abstract}

Keywords: Villagers; Citizen; Life circles

Publication date: April, 2020

Publication online: 30 April, 2020

Corresponding author: Yueping Hu, 13688366799@ sina.cn

\section{Introduction}

Under the reform and opening up, China has been undergoing urbanization rapidly, reaching to $60 \%$ in 2018. Among them, the urbanization rate of the longterm population in Chengdu is more than $70 \%$, while the urbanization rate of the registered population is more than $60 \%$. The more frequent the residents flow between urban and rural areas, the more obvious the phenomenon of counter-urbanization would be. Under the emergence of city diseases, rural landing reform and the improvement of urban and rural energy infrastructure, the trend of shifting capital to the countryside, entertainment to the countryside, retirement to the countryside, and industry to the countryside has accelerated. Habits such as going to towns, having bilateral trips on the weekend, and bilateral living are prevailing. However, the urbanrural contradiction brought about by the inertia of the urban-rural dual system over the past few decades should also be paid attention to. Issues such as price increase, consumption upgrade, talent loss have been highlighted, although they are the necessary processing issues during urbanization and counter-urbanization. It may be resolved after finishing urbanization, but it may take the youth or childhood of a generation. Therefore, this article quotes the concept of "rural circles of life" based on the suburban areas of metropolitan areas under the background of urban-rural integration, and studies the theoretical support and methods for building a rural circle of life to guide the rural development of plain metropolitan areas.

\section{Content}

\subsection{Concept of Life Circles}

The term "Life Circles" originates from the concept of "wide life circle" proposed by Japan's "Third Comprehensive Developing Plan" in 1965, which is based on comprehensive urbanization and the construction of peripheral urbanization in combination with central urban areas. They first proposed the daily life circle, and then successively proposed "local life circle" and "settling circle", in which the settling circle is the area with the basic public facilities required by residents in daily life ${ }^{[1]}$. The concept of the life circle was proposed to support the balanced urbanrural development and the basic public services in the 
urbanization of the whole region. After the concept was introduced into China, it combined both the characteristics of urban and rural areas and evolved into a circle of community life as well as a circle of rural life. It is based on the distribution of basic services required by people within certain activities.

If you trace the origin of the life circle, the range of tribal activities in the primitive society is the original form of the life circle. The rural administrative divisions formed by the agricultural society since the Tang and Song Dynasties for rule are exactly help develop the life circle. For example, in the traditional agricultural society, the market towns (market gatherings) spontaneously were formed for the needs of daily life. Villagers spontaneously gather goods and exchange information according to the agreed division of functions and time in marketing towns. For this phenomenon, American scholar Shi Jianya called it the "basic market community" in his research on China's rural market and social structure before $1948^{[2]}$. In modern times, under the influence of rapid urbanization, the administrative dividing management that originally took the town as a unit or multiple clusters cannot adapt to the needs of rural residents. Therefore, it is necessary to study a new type of urban-rural management "rural life circle" with cultural heritage.

\subsection{Analysis on the concept of rural life circle}

From the point of the source of the word, "hometown" in the Oracle bones is, meaning that two people sit opposite to each other, eating and communicating together. In primitive times, it is hard to eat alone. People who can sit and dine together at tables are not just ordinary friends but must be very familiar. Therefore, we believe that the original meaning of "hometown" refers to the scene formed in communicating activities between acquaintances.

From the perspective of administrative division, rural villages in the agricultural society are the products of administrative division since the Tang and Song Dynasties. They are a joint name for many village colonies with homogeneous culture and close interpersonal relationships. Combining with the cognition of traditional rural life, the nature of the rural life circle is based on blood relation, affinity, and geography with familiar friends, self-sufficient agricultural civilization system of equality and mutual assistance was created ${ }^{[3]}$.

However, because of the ecological damage by industrial civilization and the decline of agricultural civilization, the concept of rural life circles is also changing in the new era of ecological civilization. That is, in order to achieve high-quality development and efficient governance, based on the premise of adapting to the conditions of land and space development, rural residents build a space network that meets the need of various daily activities at different times by integrating many issues such as ecology, culture, industry, living environment and people's livelihood facilities related to the residents' production and life, forming harmony between people and nature, people and people, and people with the society.

\section{Supporting theory}

\subsection{Conclusion of the existing researches}

Compared with the life circles in urban communities that have already received the standard design and the constructing plans in various places, the research on the rural life circles in China is still in the theoretical and methodological research with few practical applications. The direction of the research mainly focuses on the delimitation of the life circle, hierarchical system, public facilities, etc. The research on the division of the scope includes the POI mobile phone GPS, time measuring algorithm and administrative division. As for the study of the level setting, there are "rule of the thirds" and "rule of the fourths" according to the scope and service level, such as primary life circle, basic life circle and daily circle; and the different scopes and levels correspond to the public facilities that meet the villagers' living needs. Generally speaking, the current research mainly regards the rural life circle as a public service circle, which is an extension of the existing balanced public service ${ }^{[4]}$. The aim is to urbanize the rural areas and form a market for consumption and the labour. Obviously, the current research is limited and too constrained in the allocation of public facilities for people's livelihood, while ignoring the overall demands of rural residents in "Developing in high quality and high efficiency as well as adhering to ecological protection first".

\subsection{Basic supporting theory}

The aim of constructing a rural life circle is to achieve high-quality development, high-quality life, highlyefficient governance and an ecologically livable village in the new era. The basis of high-quality development is the construction of modern material space, and the higher pursuit is a better social environment, 
such as a business environment, an environment for innovation full of rule of laws. The criteria for judging the quality of life are those who are benevolent and those who are wise, as well as those who have the most power to speak in the local area. Human needs define standards for quality. Highly-efficient governance is the government' pursuit and the residents' demands. Traditional administrative governance methods have always been inefficient in rural areas. The development of rural areas for thousands of years shows that rural affairs matter. Therefore, the theoretical foundation for building a rural life circle can rely on the theory of Creating Social Space, Maslow's Theory of Demand, and the Social Governance Theory.

\section{Constructing strategies}

\subsection{Analyze people and activities, and reconstruct the system of spatial activities in different periods}

The basic issues making up the rural life circle are the life, daily activities and space. The main subjects of life are villagers, citizens, urbanized villagers and local citizens. Villagers' daily activities are mainly based on the daily routine schedule of traditional agricultural society, which is roughly divided into eating and sleeping, laboring, having activities freely and doing housework, whose time ratio is roughly $4: 3: 2: 1$, on every single day, and on a quarterly climate cycle. Citizens' daily activities are mainly based on the "rest after five days' work" and "from nine to five" working system to arrange activities in the countryside, roughly divided into business days to the countryside and leisure days to the countryside, whose time cycle is seven days. The spatial carrier of the life circle is roughly divided into producing space, living space and ecological space, and its spatial scope is mainly based on the villagers' regular outings.

In terms of space, according to villagers' habits of self-sufficiency, no overnight stay, no food and slow transportation, the best region of rural life should be within 15 minutes on foot or by bike. In this region, a relatively stable industrial division of labor can be formed economically, such as the traditional Chinese "one-four-seven, two-five-eight, three-nine-nine" division of labour. They can form relatively close and densely distributed relatives and peer friends.

\subsection{Coordinate space of elements and foster a living spatial system of clear main functions}

A mature rural life circle should clearly define the attributes such as scope, hierarchy, and function. The scope of the life circle can be defined according to many existing research methods, and it can overcome the limits of administrative divisions, but it must be coordinated with the spatial boundaries of the village community. After all, natural villages are the smallest and the most stable and collective economical and residential communities. The division of the life circle can be based on the administrative governance capabilities and local geographical structure. Two levels are more appropriate than three levels. Too many levels are likely to cause administrative conflicts. The strategy of main function is a common way for spatial planning to adopt the "zoning + guiding" governance in rural areas. It is usually called a functional unit or a developmental unit. Within this policy, the rural life circles can also learn from the core industries, derivatives of the industrial ecosystem, the composition of industries and supporting industries, which are based on the main planning logic of constructing a "subject + extension" system.

A complete rural life circle also needs to take the ecology, culture, industry, living environment, and people's livelihood facilities into account, and properly deals with the relationship between protection and development. It is not advisable to lose each other and rely on any simple facility and policy. For the elements that make up the life circle, ecologists should pay attention to the protection and management of landscapes, forests, fields, lakes and grasses, cultural staff should pay attention to rural culture such as rural diet, leisure, farming, trade, belief, etc., and businessmen should pay attention to integrating agricultural, commercial and cultural tourism. The living environment includes the comprehensive renovation of both the above ground and underground, private courtyards, software and hardware, and people's living facilities such as cultural, educational, physical, and health care.

\subsection{Build a living center and create an "operating center + connecting network" social communicating system}

The countryside near the suburbs of China's 
metropolitan area is a highly interconnected area between urban and rural areas with dual activities between urban and rural areas. The biggest contradiction in life is the space-time conflict between villagers and citizens in their living habits. Villagers live according to the lunar calendar and weather conditions, while citizens live according to the weekly calendar of resting after 5 days' work, which is essentially a conflict in the time arrangement. Therefore, it is necessary to design a converter to coordinate the relationship between them, similar to the role of a converter between alternating current and direct current. This converter is like the nerve center, information hub or soul space of the rural life circle, so that various daily activities in various spaces in the circle can find the corresponding connecting point in the converter, and get information feedback or dynamic feedback.

The shape and location of the living center is not only in the constructing area, but also the ecological conservation area and agricultural developing area. The purpose is to focus on the the villagers' and citizens' living activities such as leisure, fitness, gathering, display, performing, festivals, sacrifices and so on. In the village, you can rely on the shrine, school, home for the elderly, theater, village committee, etc; In the ecological area, you can rely on forests, rivers and lakes, ancient trees, etc; In the agricultural area, you can rely on agricultural parks, trenches, ponds, etc.

The aim of constructing a rural life circle is to promote the integration of urban and rural society, and promote the cultivation of relatively stable and regular habits among villagers and citizens in the circle. Therefore, you can explore the in-depth cooperation of "heart-to-heart, hand-in-hand" in the business center of the living center and urban community center. One is to create a cultural atmosphere with the same roots and different styles through soft power such as business configuration and behaviour guidance. The second aim is to make villagers and citizens in the rural life circle form a sense of gain and accomplishment with the policies such as consumption subsidies and individual tax subsidies, or rigid consumption policies. Through these two soft and hard operating measures, they can contribute to the sustainable development of the living center and even the ecosystem of the rural life circle.

\section{Conclusion}

The construction of the rural life circle is a reflection on the current rural development dominated by material spatial construction, and it is also a response to the loss of population during urbanization and the impact of foreign culture under counter-urbanization. In this regard, we must think about how rural societies respond to the development of post-industrial societies, and try to promote the harmonious coexistence of various types of people in rural communities, the integration and coexistence of various resources, as well as the simultaneous development of humanities and nature through the revival and construction of new rural life circles. Through constructing a mainstream system based on analysis of various types of residents in rural areas and their rules of activity, a main living spatial system will be built by coordinating the spatial elements that constituting a life circle, thus the social interaction in the rural life circle will be undergone by creating a "spatial center".

\section{References}

[1] Xiao ZP, Chai YW, Zhang Y. Review on the Progress of Domestic and Foreign Planning Research and Practice on Life Circles[J]. Planner, 2014, 30 (10): 89-95.

[2] Zhang SF. Research on the Consolidation of Rural Settlements based on Life Circles [D]. Tsinghua University, 2013.

[3] Yang S, Yang HN, Ji ZM, et al. Reorganizing Mechanism of Rural Residents' Life Circle under the Rapid Urbanization: A case study of Kunshan Qunyi Community[J]. Geographic Research, 2019, 38 (1): 119-132.

[4] Yu YF. Planning from Traditional Residents' Area to Community Life Circle [J]. Urban Planning, 2019, 43 (5): $17-$ 22. 